\title{
Bandwidth, Efficiency and Directivity Enhancement of Printed Antenna Performance Using Planar Circularly Symmetric EBGs
}

\author{
N. Llombart ${ }^{1}$, A. Neto ${ }^{1}$, G. Gerini ${ }^{1}$, P. De Maagt ${ }^{2}$ \\ ${ }^{1}$ TNO Defense, Security and Safety, TNO, Den Haag 2597 AK, The Netherlands \\ E-Mail: nuria.llombartjuan, andrea.neto, giampiero.gerini@tno.nl \\ 2 Electromagnetics Division, European Space Agency, 2200 AG, Noordwijk, The Netherlands \\ E-mail: Peter.de.Maagt@esa.int
}

\begin{abstract}
Planar Circularly Symmetric (PCS) Electromagnetic Band-Gap (EBG) substrates have been recently proposed to suppress the surface waves in printed technology [1] [2]. The major advantage of the PCS-EBGs with respect to structures based on vertical pins [3] [4] is the fabrication simplicity since the dielectric slabs do not need to be perforated. With respect to other planar type of EBG [5], an advantage is that the surface waves launched by a central source are reduced equally in all radial directions. The motivation of this contribution is to design and measure a prototype which demonstrates the suppression of surface waves by a PCS-EBG of a single antenna printed on a dielectric substrate. Thanks to the presence of the PCS-EBG around the antenna, we obtain an enhancement of the bandwidth, efficiency and directivity performances. The study will present some design considerations for the PCS-EBG itself, and then will proceed with the manufactured and measured antennas. The results explicitly show the advantages in terms of bandwidth and radiation pattern of the proposed EBG substrate. A bandwidth of $20 \%$ is achieved without significant surface wave losses.
\end{abstract}

\section{INTRODUCTION}

Electromagnetic band-gap (EBGs) materials [6] are being proposed for solving a wide variety of E.M. problems [3][5]. In this paper a procedure to design artificial substrates that impede surface wave propagation over wide bands, and are suited to host antennas with bandwidths up to $20 \%$, is presented. The planar structures that we consider are circularly symmetric and radially periodic and are aimed to the suppression of the $T M_{0}$ mode of the grounded slab. The key characteristics of PCS-EBGs are the following: they are simple to manufacture since they do not present vertical via holes or pins, they do present the same band-gap properties for different directions of propagation, and, finally, PCS-EBGs can be designed starting from a $2 \mathrm{D}$ equivalent geometry with a $1 \mathrm{D}$ periodicity. The $2 \mathrm{D}$ geometry yields a very good first order estimation of the overall performances of the relevant $3 \mathrm{D}$ geometry, while reducing the numerical effort.

In order to quantify the improvement of the PCS-EBG on the performances of a central antenna, a panel composed of six printed antennas has been built (Fig. 7). Four antennas are simple printed antennas whose geometry is explained in section II. The other two antennas are surrounded by a PCSEBG consisting of two or three rings. The geometry and design issues of a PCS-EBG are explained in section III. The improved performances can be seen in terms of increase of bandwidth, reduction of the mutual coupling between the antennas, cleaner radiation pattern due to less edge effects and

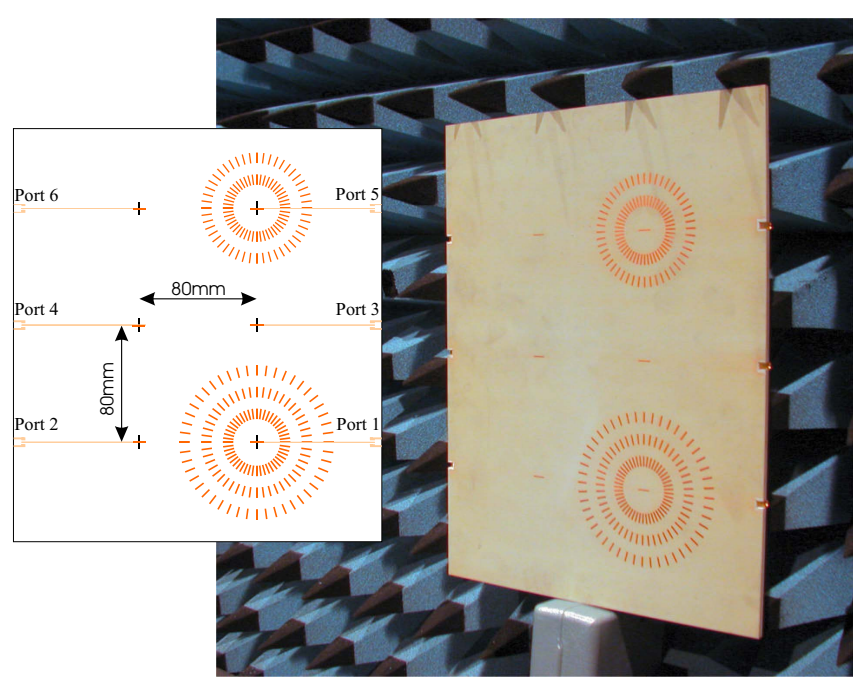

Fig. 1. Panel with six antennas located in a square grid of $80 \mathrm{~mm}$.

improvement of the gain. The measures of the S-parameters are shown in section IV, and finally the radiation patters and gain are shown in section $\mathrm{V}$.

\section{Micro-Strip Excited, Slot Coupled Dipole}

The central antenna, in absence of the EBG, is shown in Fig. 2. It consists of two dielectric slabs with the same dielectric constant $\epsilon_{r}$ and different heights $h$ and $h_{m}$ divided by a ground plane. There is a slot etched in the ground plane of dimensions $l_{s}$ and $w_{s}$. The slot is coupled to a orthogonal dipole $\left(l_{d}\right.$ and $\left.w_{d}\right)$ located on the top of the upper dielectric slab $h$. Finally, the structure is excited via a micro-strip whose extension beyond the slot crossing is $l_{\text {stub }}$ while its width is $w_{m}$. The micro-strip is printed on the other side of the lower dielectric slab $h_{m}$.

Printed antennas usually use low dielectric constants so that there is no significant excitation of surface waves. However, when using slot coupled antennas, this comes at the cost of a low front to back ratio which often implies the presence of a backing reflector. Such backing reflector will eventually allow the propagation of parallel plate waveguide modes, which will have to be eliminated somehow, for instance by using EBGs or cavity backing. The key advantages of antennas printed on moderately high dielectric constant slabs $\left(\epsilon_{r}=10\right)$ would be 


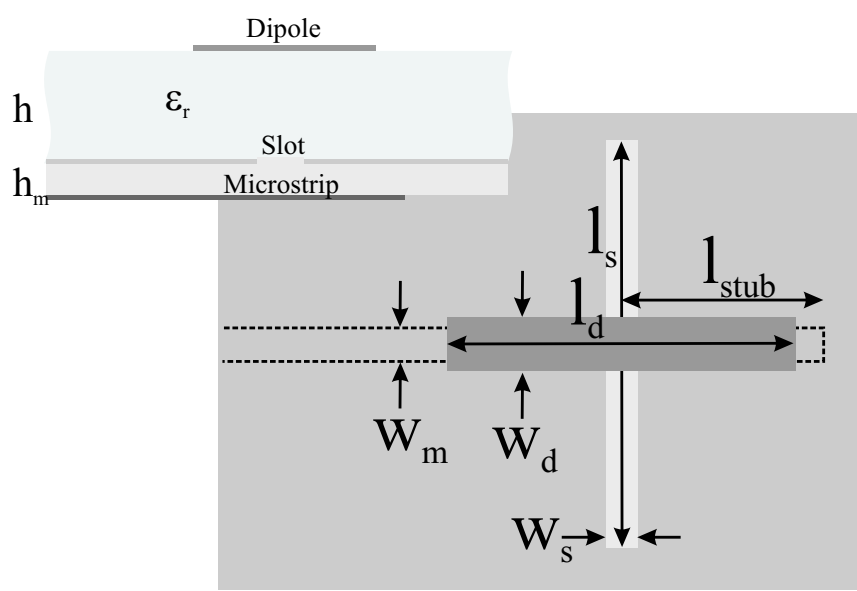

Fig. 2. Side and top view of the printed antenna: Slot $\left(l_{s}, w_{s}\right)$ coupled to a dipole $\left(l_{d}, w_{d}\right)$ and excited via micro-strip $\left(l_{s t u b}, w_{m}\right)$.

the simplification of the feeding network since the front to back ratio would result higher. In [7] was demonstrated that the impedance bandwidth of a printed antenna is maximum for slab of thickness close to the radiation peak $\left(h \approx \lambda_{d} / 4\right)$, which corresponds to the excitation of the second slab mode, i.e. $T E_{1}$. Even if the substrate we considered in this paper has such a thickness that only the $T M_{0}$ mode is in propagation ( $\left.h \approx 0.22 \lambda_{d} / 4\right)$, the surface wave results extremely strong excited. In case of moderately high dielectric constant slabs $\left(\epsilon_{r}=10\right)$, more than $70 \%$ of the total power is launched into the first TM surface wave [8]. As consequence these antennas are not suitable unless an ad hoc procedure to block surface waves is introduced.

\section{DESIGN OF THE PCS-EBG}

The PCS-EBG structure, shown in Fig. 3, is arranged in a circularly symmetric form to provide an effective control of the surface waves generated by a central antenna in all radial directions. The radial positions and lengths of the dipoles, $\left(l_{g}\right.$, $d)$, are designed using a two dimensional problem in order to obtain a band-gap of the $T M_{0}$ mode in the frequency band of interest. The dispersion diagrams of 2D-EBG were calculated in [9]. The procedure included the set up of an integral equation enforcing the vanishing of the electric field (EFIE) on the metallic loadings. In Fig. 4 the real and imaginary parts of the normalized solutions, $k / k_{0}$, of the dispersion equations pertinent to a grounded dielectric slab $\left(\epsilon_{r}=10, h=3.81 \mathrm{~mm}\right)$ loaded by a strip grating $\left(l_{g}=6.6 \mathrm{~mm}, d=13.7 \mathrm{~mm}\right)$ are shown.

In [1], PCS-EBGs were used to suppress the surface waves launched by a symmetric TM source to improve the efficiency of such antennas. A symmetric TM source generates only a pure TM field with the electric field entirely polarized along $\rho$. Continuous rings were used to stop the surface wave propagation. When the source is not TM symmetric, it generates electric field components in both, $\rho$ and $\phi$, directions. If one was simply to use the continuous rings, they would support azimuthal electric currents that can lead to strong resonances. Such strong resonances will then be responsible for a significant alteration of the input impedance and thus a bandwidth reduction. However it can be demonstrated that the electric field associated to the TM surface waves, for large

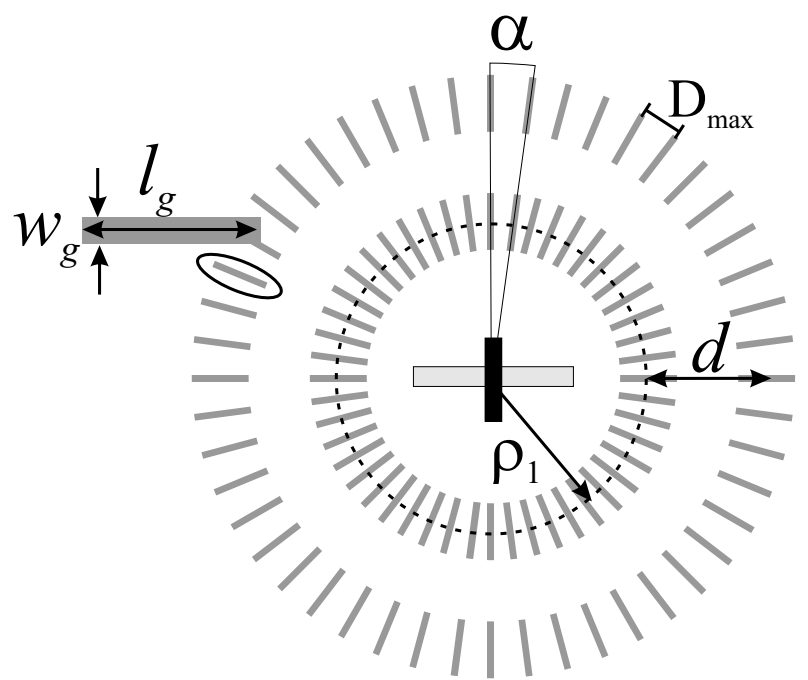

Fig. 3. Geometrical parameters of a PCS-EBG.

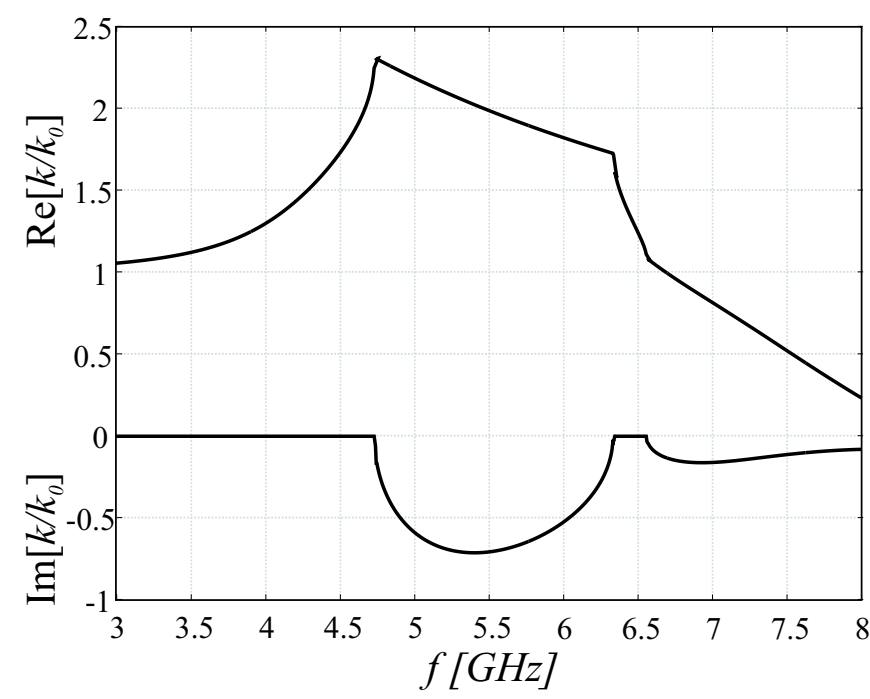

Fig. 4. Dispersion diagram for a grounded slab with parameters $\epsilon_{r}=10$ and $h=3.81 \mathrm{~mm}$ loaded by a strip grating of $d=13.7 \mathrm{~mm}$ and $l_{g}=6.6 \mathrm{~mm}$

values of $\rho$, has an electric field with no $\phi$ component. Thus, a simple modification of the PCS-EBG configurations that resorts to dipoles oriented in the radial direction around the source can be applied. The radial dipoles will only act on the $\rho$ component of the electric currents (or of the electric fields), so will not introduce additional resonances.

The influence of the width of the dipoles $w_{g}$ is small. Instead, the angle at which the dipoles are placed $\alpha$ (it defines the number of dipoles per ring), has to be chosen so that the $T M_{0}$ mode still experiences a continuous surface (like continuous rings). In terms of array theory this implies that each wave front, that has normal incidence, must encounter elements that are sampled in $\phi$, at least, at the Nyquist frequency: $D_{\max } \leq \frac{\lambda_{T M 0}}{2} \leq \frac{\pi}{k_{T M 0}}$ where $k_{T M 0}$ is the propagation constant of the $T M_{0}$ mode of the grounded slab. The parameter $\rho_{1}$, instead, is selected to be half of the surface wave wavelength, $\lambda_{T M 0}$ :

$$
\rho_{1}=\frac{\lambda_{T M 0}}{2}
$$




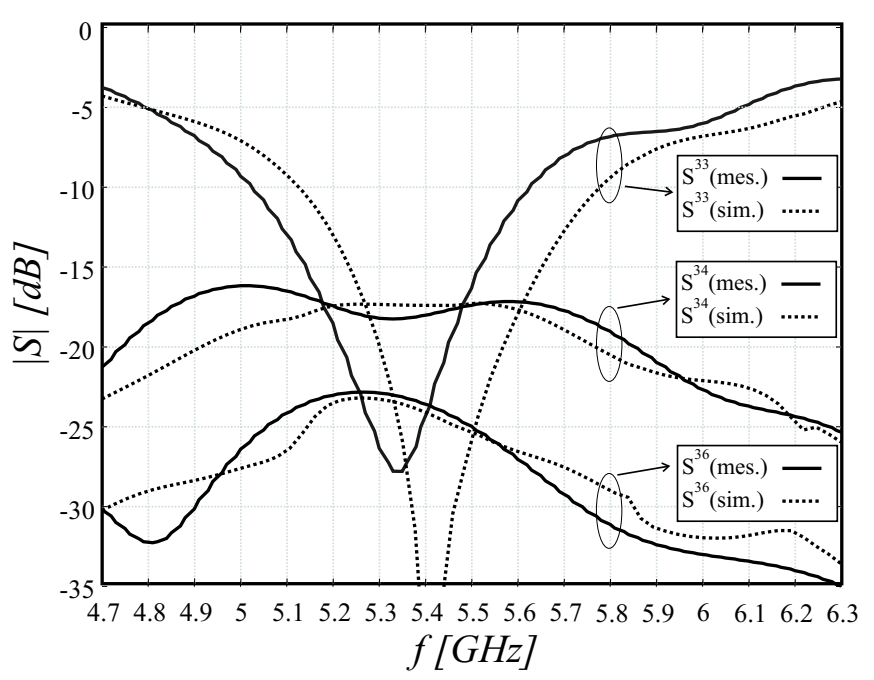

Fig. 5. S-parameters pertinent to the antennas without EBG

in order to enhance the radiation bandwidth. In this condition, the surface waves launched by the central source are cancelled by the ones reflected from the EBG. Thus only the spectral components of the fields contributing to radiation are significant in the source. This aspect will be further investigated.

\section{S-PARAMETERS}

Fig. 5 shows the S-parameters pertinent to the antennas without the PCS-EBG around them. The measurements are compared with the simulations done using Ansoft Designer. One can first note that the single element impedance bandwidth $\left(S_{33}<-10 \mathrm{~dB}\right)$ is in the order of $12 \%$. This bandwidth is not useful because this antenna has a very poor efficiency of around $40 \%$ due to the strong excitation of surface waves. The $S_{34}$, case in which the antennas are situated at a distance of $80 \mathrm{~mm}\left(1.45 \lambda_{0}\right.$ at the central frequency) in the maximum coupling direction, is around $-17 \mathrm{~dB}$. The second parameter $S_{36}$ is around $-23 \mathrm{~dB}$ and corresponds to case where the antennas are placed at $45^{\circ}$ one to the other. For both these configurations the coupling is essentially due to the $T M_{0}$ surface wave.

Fig. 6 shows the S-Parameters related to the antenna with the two rings EBG surrounding it. The $S_{55}$ parameter of the antenna surrounded by the EBG is improved, thanks to the appropriate selection of $\rho_{1}$, so that the impedance bandwidth is about $20 \%$. Most important, there is also a significant reduction of the coupling between the two antennas. Both the $S_{54}$ and $S_{56}$ parameters are reduced in around $10 \mathrm{dBs}$ arriving to coupling levels dominated by the space wave. These results show that as far as impedance bandwidth is concerned PCSEBG may be tuned to achieve $20 \%$ bandwidth without surface wave excitation.

Finally, the results pertinent to the EBG based on three rings are not discussed here. It should be mentioned that the third ring does not significantly improve the isolation between the antennas, because thanks to the attenuation in the band-gap (imaginary part of $k$ in the dispersion diagram, fig. 4) two rings are sufficient to suppress the surface wave.

\section{RADiATION PATTERNS}

In order to perform the measurements and obtain a fair comparison between the performances of the different antenna

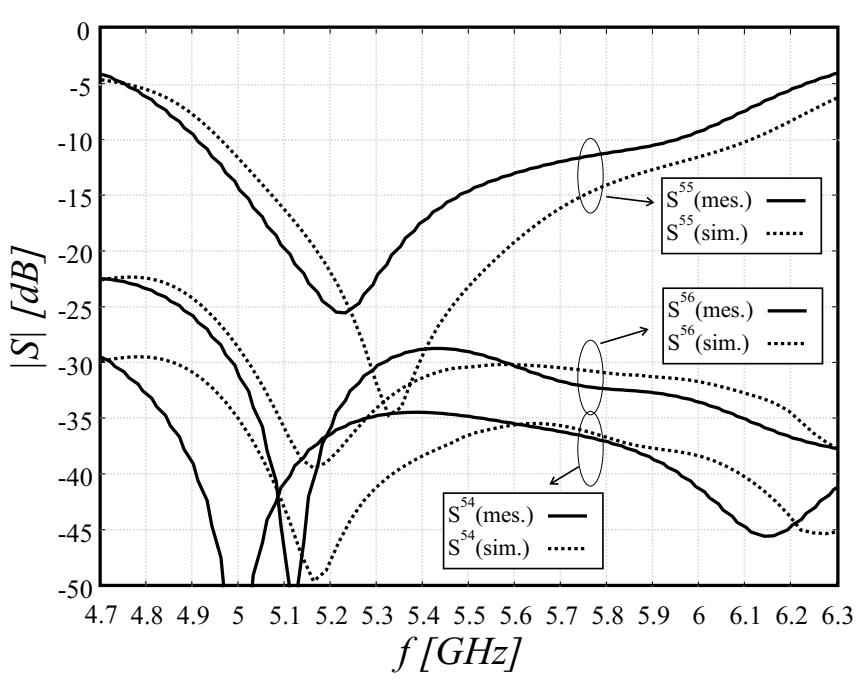

Fig. 6. S-parameters pertinent to to the antenna surrounded by 2 rings of dipoles

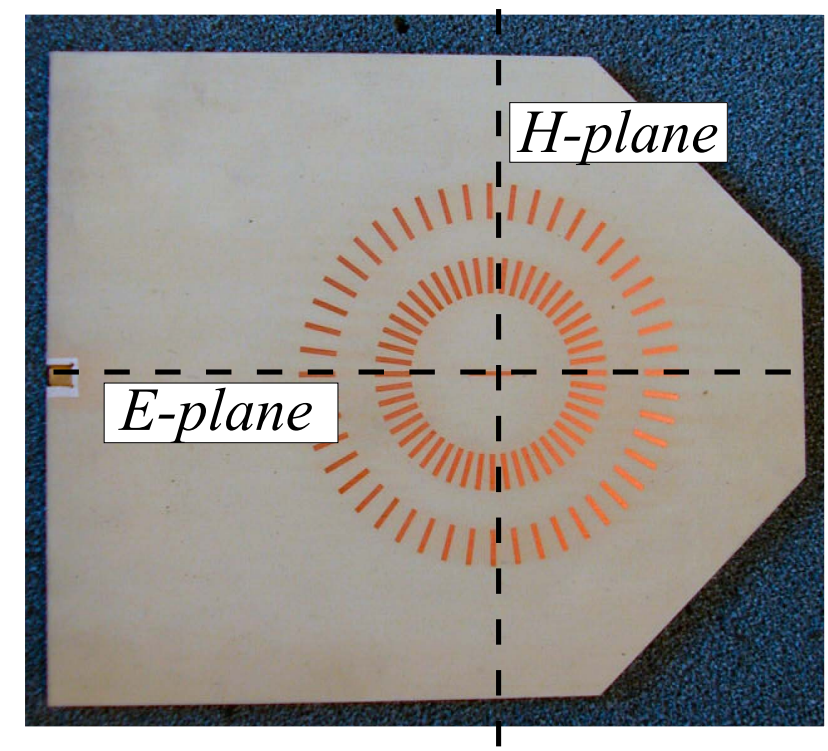

Fig. 7. Panel portion containing the antenna surrounded by two rings

configurations (no EBG and two rings EBG), the panel has been cut in portions of exactly the same dimension. A photograph of one of these panels is shown in Fig. 7. Also the E-and $\mathrm{H}$-plane cuts are shown in the same figure. The measurements were then performed in the cylindrical near field scanning facility at TNO.

Fig. 8 and Fig. 9 show the co-polar normalized radiation patterns in the H-plane and E-plane mesured at the central frequency $(\mathrm{f}=5.4 \mathrm{GHz})$ for the two antennas considered. Observing the H-plane first, Fig. 8, one can notice a very significant difference between the $3 \mathrm{~dB}$ angle obtained with and without EBGs. Especially in absence of EBGs, the surface wave diffraction is expected to heavily affect the actual radiation patterns. Fig. 9 shows the measured radiation patterns pertinent to the E-plane, which is the plane where the surface waves is strongest excited. In this case the patterns are in general not symmetric, due to the asymmetry of the panel cut. Most importantly the impact of surface wave diffraction on the single antenna pattern is significant with a first deep null 


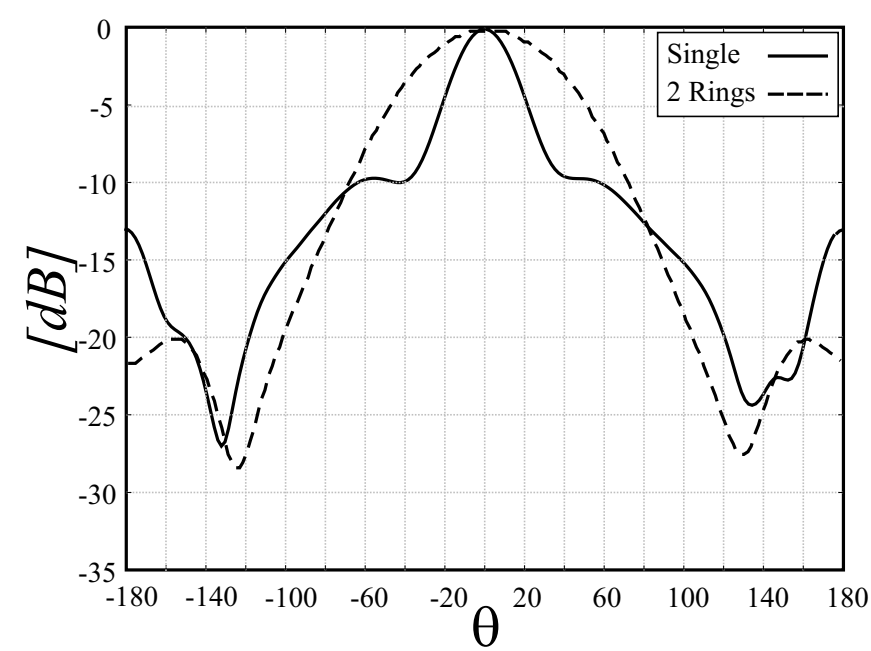

Fig. 8. H-plane radiation pattern measured at the central frequency for the single antenna and the PCS-EBG antenna with two rings.

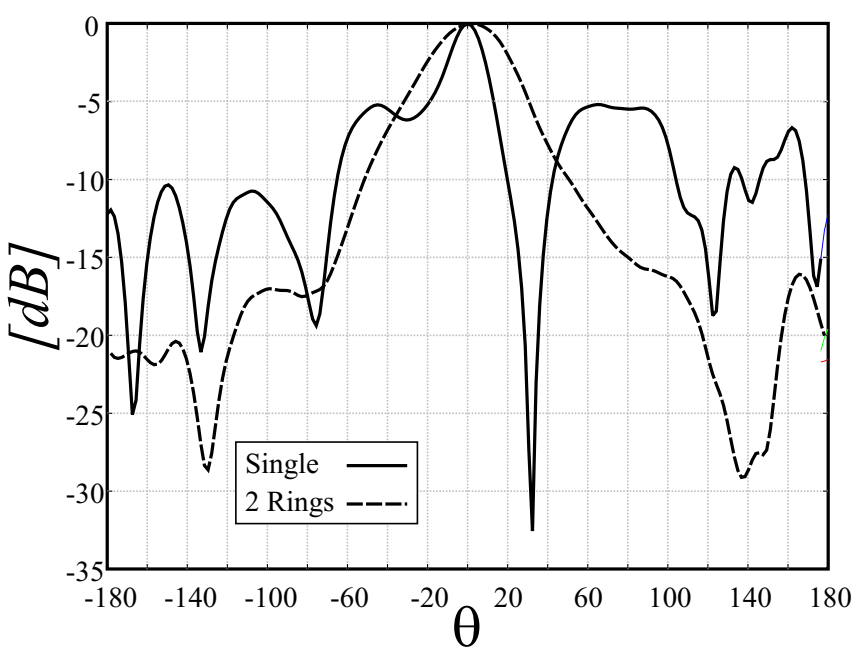

Fig. 9. E-plane radiation pattern measured at the central frequency for the single antenna and the PCS-EBG antenna with two rings.

well inside the $3 \mathrm{~dB}$ radiation pattern region.

The same antennas have been simulated with Ansoft Designer which considers an infinite substrate (no edge effects). The gain given by this tool can be interpreted as a measure of the radiation efficiency of the antenna, and is shown in Fig. 10. In the presented designs, the antenna without EBG has a very low gain in the order of $1 \mathrm{~dB}$ (due to the fact that more than $60 \%$ of the power is launched into the $T M_{0}$ surface wave and the diffraction at the edge of the substrate is not accounted for). However even if real gain, including edge effects, turns to be higher, it is not controllable thus cannot be used. When the antenna is simulated with the PCSEBG with 2 rings around it, a significant gain improvement of around $8.5 \mathrm{dBs}$ is observed. Already with 2 rings a surface wave efficiency of $90 \%$ is obtained, which corresponds to $3 \mathrm{dBs}$ of gain improvement. The additional gain improvement is associated to a larger effective area of the antenna.

\section{CONCLUSION}

In this paper the use of purely planar circularly symmetric periodic metallic loadings has been proposed to impede the propagation of the $T M_{0}$ surface wave in planar printed

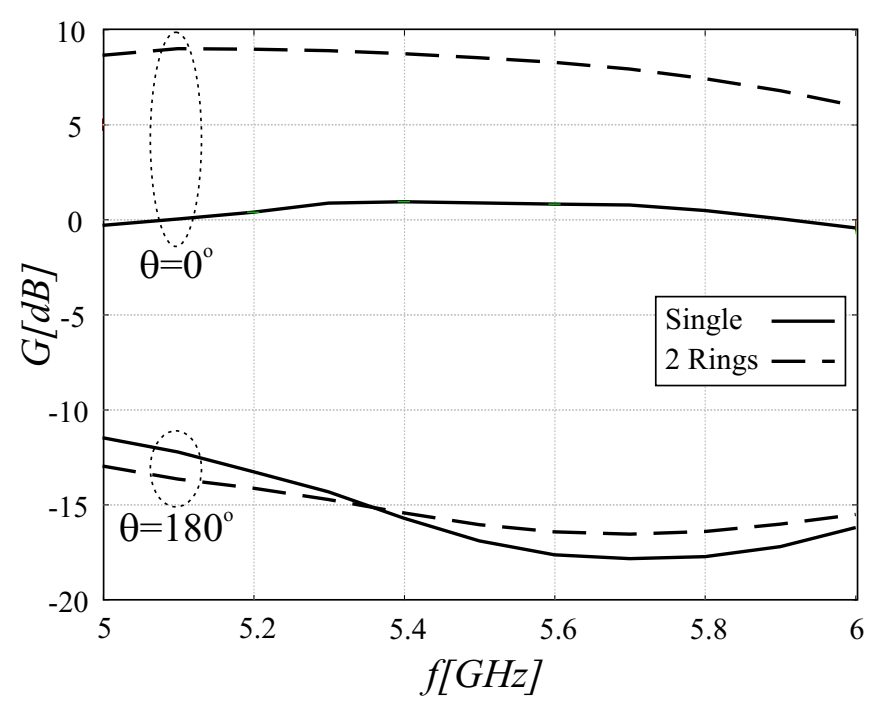

Fig. 10. Simulated gain at broadside and backside for the single antenna and the PCS-EBG antenna with two rings.

structures. The main advantage of the design simplicity is that the performances of the ensemble of antenna and EBG can be easily optimized to meet specific requirements. An antenna with $20 \%$ of bandwidth has been presented which does not suffer from surface wave effects. The radius of the PCS-EBG is half of the surface wave length, $\lambda_{T M 0}$, for maximum radiation bandwidth. This will be demonstrated in further publication.

\section{REFERENCES}

[1] N. LLombart, A. Neto, G. Gerini and P.J. de Maagt "Planar Circularly Symmetric EBG structures: Design and Analysis" in European Microwave Week, Amsterdam, The Netherlands, October 2004.

[2] M. Llombart, A. Neto, G. Gerini and P.J. de Maagt "Enhanced Antenna Performances Using Planar Circularly Symmetric EBGs" in 2005 IEEE AP-S Ineternation Symposium, Washington DC, 3-8 July 2005.

[3] P. de Maagt, R. Gonzalo, Y.C. Vardaxoglou and J.M. Baracco, "Electromagnetic bandgap antennas and components for microwave and (Sub)millimeter wave applications" in IEEE Transactions on Antennas and Propagation, vol. 51, no. 10, pp. 2667-2677, October 2003.

[4] D. Sievenpiper, L. Zhang, R.F. Broas, N.G. Alexopolous and E. Yablonovitch, "High-impedance Electromagnetic Surfaces with a Forbidded Frequency Band", in IEEE Transactions on Microwave Theory and Techniques, vol. 47, no. 11, pp. 2059-2074, November 1999.

[5] R. Coccioli, F-R. Yang, K-P Ma and T. Itoh, "Aperture-coupled Patch antennas on UC-PBG", in IEEE Transactions on Microwave Theory and Techniques, vol. 47, no. 11, pp. 2123-2130, November 1999.

[6] E. Yablonovitch, "Inhibited Spontaneous Emission in Solid State Physics and Electronics", in Physical Review Letters, vol. 58, no. 20, pp. 20592062, May 1987.

[7] N. G. Alexopoulus, P. B. Katehi and D. B. Rutledge "Substrate Optimization for Integrated Circuit Antennas", in IEEE Transactions on Microwave Theory and Techniques, vol. 31, no. 7, pp. 550-557, July 1983.

[8] S. F. Mahmoud, Y. M. M. Antar, H. F. Hammad and A. P. Freundorfer "Theoretical Considerations in the Optimization of Surface Waves on a Planar Structure", IEEE Transactions on Antennas and Propagation, vol. 52, no. 8, pp. 2057-2063, August 2004.

[9] N. LLombart, A. Neto, G. Gerini and P.J. de Maagt "Periodic Structures excited by non periodic sources" in 27-th ESTEC workshop on innovative periodic structures, Santiago de Compostela,Spain, 10-13 March 2004. 